\title{
Effective easy-axis anisotropy of the two-sublattice single-chain magnet with twisted easy planes
}

\author{
M. S. Shustin ${ }^{1}$, M. N. Potkina ${ }^{2,3,4}$ \\ ${ }^{1}$ L. V. Kirensky Institute of Physics, 660036, Krasnoyarsk, Russia \\ ${ }^{2}$ ITMO University, Kronverkskiy, 49, St. Petersburg, 197101, Russia \\ ${ }^{3}$ St. Petersburg State University, St. Petersburg, 198504, Russia \\ ${ }^{4}$ Science Institute and Faculty of Physical Sciences, Univ. of Iceland 107 Reykjavík, Iceland \\ mshustin@yandex.ru,potkina.maria@yandex.ru
}

PACS 75.30.Gw, 75.47.-m, 75.10.Dg

DOI 10.17586/2220-8054-2020-11-6-659-665

\begin{abstract}
An analytical solution for the spin-wave spectrum of the two-sublattice 1D magnet with $S_{A}=S_{B}=1$ and twisting easy planes has been obtained. Such planes are mutually twisted by an angle $\varphi$ relative to each other. For the case of mutually orthogonal easy planes $\varphi=\pi / 2$, the spectrum vs. quasi-momentum dependence has been compared with that of an easy-axis magnet with the easy axis aligned along the line of intersection of the planes. An analogy of the spectra of the models has been shown, indicating the possibility of the effective easy axis anisotropy in easy-plane two-sublattice single-chain magnets.
\end{abstract}

Keywords: single-chain magnets, magnonic spectrum, strong single-ion anisotropy.

Received: 18 October 2020

Revised: 10 November 2020

\section{Introduction}

In recent years, strongly anisotropic single-chain magnets (SCM) have attracted considerable attention in view of their promising use in magnetic memory and spintronics devices [1]. From a fundamental point of view, SCMs are of interest as low-dimensional magnetic materials with developed spin fluctuations [2-4]. To date, several tens of single-chain magnets have been synthesized with different properties. From the prospects for spintronics, compounds supporting radiation-induced long-lived magnetic excitations are of considerable interest $[1,5,6]$. Such compounds include the four-sublattice SCM catena - $\left[\mathrm{Fe}^{I I}\left(\mathrm{ClO}_{4}\right)_{2} \mathrm{Fe}^{I I I}(\text { bpca })_{2}\right]\left(\mathrm{ClO}_{4}\right)$ [7-9] with twisting and mutually ortoghonal easy planes. Due to the different orientations of the above easy planes, the effective easy-axis anisotropy is induced and microscopic magnetic domains with sharp domain walls are created [7,9]. On the other hand, the presence of the easy-plane single-ion anisotropy induces strong spin fluctuations [10,11] which have to be taken into account for consecutive analysis of experimental data $[8,12]$. One should expect formation of similar easy-axis anisotropy in twosublattice single-chain magnets with twisted directions of the easy planes. In this work, this effect is considered on the basis of analytical calculations of the spin-wave spectra of spin-one two-sublattice 1D magnets: an easy-plane magnet with twisted easy planes, and an easy-axis one. This problem has been solved with the use of atomic representation detailed in the works $[7,10,13]$.

\section{Spin-wave spectrum of the two-sublattice anisotropic SCMs}

The main goal of this work is to formulate a quantum spin model of easy-plane single-chain magnet, for which the emergence of a macroscopic easy-axis can be demonstrated analytically. Since single-chain magnets are lowdimensional and highly anisotropic, it is important that such a consideration correctly takes into account strong anisotropy and spin fluctuations. These requirements lead to a certain compromise. On the one hand, the requirement of simple and analytical consideration leads to a model with a small number of sublattices and the small spin values of the sublattices. On the other hand, since the contribution of quantum fluctuations is significant for magnets with small spin moments, the theory under consideration should take into account these effects beyond the mean-field approximation. As a result, we propose a model of a two-sublattice spin-one single-chain magnet with alternating and mutually twisted on the angle $\varphi$ easy-planes. This model is considered in the generalized spin-wave approximation, beyond anisotropic mean field approximation. In this work, we consider a low-energy excitation spectrum of the model, which determines its low-temperature thermodynamics. 
Let us consider an anisotropic two-sublattice 1D magnet with sublattice spins $S_{A}=S_{B}=1$ and alternated easy planes mutually twisted on the angle $\varphi$. The Hamiltonian of the system is:

$$
\widehat{\mathscr{H}}=\sum_{f \in A}\left[\mathscr{H}_{A}^{a}\left(\vec{S}_{f}\right)-\bar{H}_{A} S_{f}^{z}\right]+\sum_{g \in B}\left[\mathscr{H}_{B}^{a}\left(\vec{S}_{g}\right)-\bar{H}_{B} S_{g}^{z}\right]-J \sum_{\langle f, g\rangle} \Delta \vec{S}_{f} \Delta \vec{S}_{g}
$$

where the symbol $\langle f, g\rangle$ means the summation over the neighboring sites, $\vec{S}_{f}$ and $\vec{S}_{g}$ are the vector operators of the spin moments of the sites $f$ and $g$ associated with $A$ and $B$ sublattices, respectively. $\Delta \vec{S}_{f}=\vec{S}_{f}-\left\langle\vec{S}_{A}\right\rangle, \Delta \vec{S}_{g}=\vec{S}_{g}-\left\langle\vec{S}_{B}\right\rangle$. The single-ion anisotropy of the ions on the $A$ and $B$ sublattices are described by operators $\mathscr{H}_{A}^{a}\left(\vec{S}_{f}\right)$ and $\mathscr{H}_{B}^{a}\left(\vec{S}_{g}\right)$, respectively:

$$
\mathscr{H}_{A}^{a}\left(\vec{S}_{f}\right)=2 D\left(S_{f}^{x}\right)^{2} ; \quad \mathscr{H}_{B}^{a}\left(\vec{S}_{g}\right)=0.5 \cdot D\left(S_{g}^{+} e^{-i \varphi}+S_{g}^{-} e^{i \varphi}\right)^{2} .
$$

where $\bar{H}_{A(B)}=H-J\left\langle S_{g(f)}^{z}\right\rangle$ are the effective magnetic fields, which contribute to the sublattices $A(B)$, respectively. In this research, we assume the external magnetic field to be zero $(H=0) . D>0$ is the parameter of easy-plane single-ion anisotropy; $J>0$ is the exchange parameter, wherein $D$ is assumed to be comparable or superior to $J$. Thus, in the following theory the single-ion contributions to the Hamiltonian (the first two terms in (1)) are considered exactly, while the fluctuation correction to the exchange interaction (the last term in (1)) is considered as a perturbation. In the expression for the Hamiltonian, we omitted the constant term, since it does not contribute to the excitation spectrum and is not interesting for the present study. We note that excitation spectrum has been assumed to be gapped, which corresponds to the existence of the short-range magnetic ordering with averages $\left\langle S_{f, g}^{z}\right\rangle$.

For what follows, it is important to note two important cases (see Fig. 1): a) $\varphi=0$ and b) $\varphi=\pi / 2$. At $\varphi=0$ the system is equivalent to the easy-plane 1D magnet (Fig. 1a) considered in [10]. Its spin-wave excitation spectrum is determined as:

$$
\omega_{\perp}(q)=\sqrt{\left(\varepsilon_{21}-J_{q}\right)^{2}-J_{q}^{2} \sin ^{2} 2 \theta} ; \quad \omega_{\|}(q)=\sqrt{\left(\varepsilon_{31}-2 \varepsilon_{31} J_{q} \sin ^{2} 2 \theta\right)} ;
$$

where

$$
\begin{aligned}
\varepsilon_{21} & =D+\sqrt{D^{2}+\bar{H}^{2}} ; & \varepsilon_{31}=2 \sqrt{D^{2}+\bar{H}^{2}} \\
\sin 2 \theta & =-D /\left(\bar{H}^{2}+D^{2}\right)^{1 / 2} ; & \cos 2 \theta=\bar{H} /\left(\bar{H}^{2}+D^{2}\right)^{1 / 2} ;
\end{aligned}
$$

$\bar{H}=\bar{H}_{A}=\bar{H}_{B} ; J_{q}=2 J \cos q$ is the Fourier-transform with quasimomentum $q$ of the exchange interaction amplitude. Note that due to introduction of two sublattices $-\pi / 2<q<\pi / 2$, and for this case, there are also branches $\omega_{\perp}(q+\pi / 2)$ and $\omega_{\|}(q+\pi / 2)$. For such a spectrum, the width of the spin-wave band is many times greater than the gap in the excitation spectrum, and there is no effective easy-axis anisotropy.

a) $\varphi=0$ :

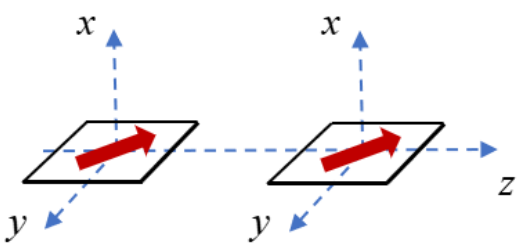

b) $\varphi=\pi / 2$ :

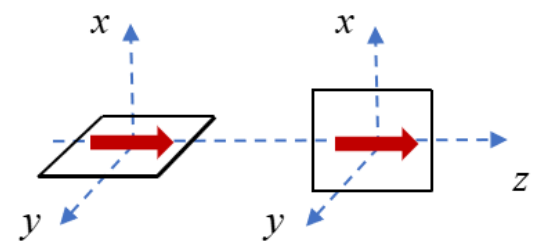

FIG. 1. Structure of the magnetic cells of the two-sublattice easy-plane 1D magnet with twisted on the angle $\varphi$ easy planes. (a) the case of $\varphi=0$ then the system is equivalent to the easy-plane 1D magnet, (b) the case of twisted easy planes, $\varphi=\pi / 2$.

At the $\varphi=\pi / 2$ case (mutually orthogonal easy-planes, Fig. 1b), we expect from the semiclassical analysis the formation of the effective easy axis. This is because the magnetic moments of two different sublattices, on the one hand, tend to lie in mutually orthogonal planes due to strong anisotropy, and, on the other hand, to be oriented parallel to each other due to exchange interaction. The only possible compromise in this case is the alignment of the moments 
along the easy-planes intersection line (the z-axis in Fig. 1). This means that the thermodynamics of the system will be analogous to that of an easy-axis magnet. Since the thermodynamics of the system is determined by the excitation spectrum, let us consider the evolution of the one with a change of $\varphi$.

In order to take into account the anisotropy exactly we use the ideology of atomic representation [13]. This representation assumes a diagonalisation of the single sites operators of the Hamiltonian (2):

$$
\left[H_{A(B)}^{a}(\vec{S})-\bar{H}_{A(B)} S^{z}\right]\left|\psi_{p}\right\rangle_{A(B)}=E_{p}\left|\psi_{p}\right\rangle_{A(B)}
$$

where energy levels $E_{p}$ ( $\left.p=1,2,3\right)$ are independent of sublattice indices. They are related with the parameters $\varepsilon_{21}=E_{2}-E_{1}$ and $\varepsilon_{31}=E_{3}-E_{1}$ introduced earlier (4). Bearing in mind that the sublattice $\mathrm{A}$ is equivalent to sublattice B at $\varphi=0$, we present expressions for single-ion states and their energies in general form as functions of $\varphi$ :

$$
\begin{array}{ll}
\left|\psi_{1}\right\rangle=e^{-i \varphi} \cos \theta|1\rangle+e^{i \varphi} \sin \theta|-1\rangle ; & E_{1}=D-\sqrt{D^{2}+\bar{H}^{2}} \\
\left|\psi_{2}\right\rangle=|0\rangle ; & E_{2}=2 D ; \\
\left|\psi_{3}\right\rangle=-e^{-i \varphi} \sin \theta|1\rangle+e^{i \varphi} \cos \theta|-1\rangle ; & E_{3}=D+\sqrt{D^{2}+\bar{H}^{2}} \\
\cos \theta=\sqrt{\frac{1}{2}\left(1+\frac{\bar{H}}{\sqrt{D^{2}+\bar{H}^{2}}}\right) ;} \quad \sin \theta=-\sqrt{\frac{1}{2}\left(1-\frac{\bar{H}}{\sqrt{D^{2}+\bar{H}^{2}}}\right)}
\end{array}
$$

Here, we used the eigenbasis $|M\rangle$ of the $S^{z}$ operator $(M= \pm 1,0)$. Following to the [7, 10, 13] let us introduce the Hubbard operators $X_{f j}^{p q}=\left|\psi_{p ; f j}\right\rangle\left\langle\psi_{q ; f j}\right|$ based on the eigenstates $\left\{\left|\psi_{p ; f j}\right\rangle\right\}$ on the cites $f$ of the $j=A, B$ sublattices. In this representation the spin operators have the form:

$$
S_{j}^{z}=\sum_{\alpha} \gamma_{\|, j}(\alpha) X_{j}^{\alpha}+\sum_{p} \Gamma_{\|, j}(p) h_{j}^{p} ; S_{j}^{+}=\sum_{\alpha} \gamma_{\perp, j}(\alpha) X_{j}^{\alpha}+\sum_{p} \Gamma_{\perp, j}(p) h_{j}^{p}
$$

Summation over $\alpha$ means summation over the root vectors $\alpha=\alpha(p, q)$ [13], which describes the transitions from the initial $\left|\psi_{p}\right\rangle$ to the final $\left|\psi_{q}\right\rangle$ single-ion eigenstate. The definition of root vectors follows from the system of equalities: $X^{p q}=X^{\alpha(p, q)}=X^{\alpha} ; X^{q p}=X^{-\alpha(p, q)}=X^{-\alpha}$. Moreover, $\gamma_{\|}(\alpha), \Gamma_{\|}(\alpha)$ and $\gamma_{\perp}(\alpha), \Gamma_{\perp}(\alpha)$ are, respectively, the longitudinal and transverse parameters of the representation of the operators $S^{z}$ and $S^{+}$in terms of the Hubbard operators:

$$
\gamma_{\|(\perp) j}(\alpha(p, q))=\left\langle\psi_{p}^{j}\left|S_{j}^{z(+)}\right| \psi_{q}^{j}\right\rangle ; \Gamma_{\|(\perp) j}(p)=\left\langle\psi_{p}^{j}\left|S_{j}^{z(+)}\right| \psi_{p}^{j}\right\rangle
$$

Let us introduce the retarded Green functions:

$$
G_{j j^{\prime}}^{\alpha \beta}\left(f-f^{\prime} ; t-t^{\prime}\right) \equiv\left\langle\left\langle X_{f j}^{\alpha}(t) \mid X_{f^{\prime} j^{\prime}}^{-\beta}\left(t^{\prime}\right)\right\rangle\right\rangle=-i \theta\left(t-t^{\prime}\right)\left\langle\left[X_{f j}^{\alpha}(t), X_{f^{\prime} j^{\prime}}^{-\beta}\left(t^{\prime}\right)\right]\right\rangle .
$$

where $X_{f j}^{\alpha}(t)$ are the Hubbard operators in the Heisenberg representation. The exact system of equations of motion for such functions is infinite [14]. However, using the Hubbard I approximation [15]: $\left\langle\left[X_{f j}^{\alpha}(t), X_{f^{\prime} j^{\prime}}^{-\beta}\left(t^{\prime}\right)\right]\right\rangle=$ $\delta_{f f^{\prime}} \delta_{j j^{\prime}} \delta_{\alpha \beta} b_{j \alpha}$ a closed system of equations for the Green's functions can be found in the frequency-quasimomentum representation. The algorithm for finding the Green's functions is described in [12]. For the considered model (1) we obtain using this approach

$$
\begin{aligned}
& G_{j \alpha ; j \beta}\left(\vec{q}, \omega_{n}\right)=\delta_{\alpha, \beta} G_{j \alpha}\left(\omega_{n}\right) b_{j}(\alpha)+ \\
& J_{q} G_{j \alpha}\left(\omega_{n}\right) G_{j \beta}\left(\omega_{n}\right) b_{j}(\alpha) b_{j}(\beta)\left\{\Delta_{\|}^{-1}\left(\vec{q}, \omega_{n}\right) \cdot 2 J_{q} u_{j}\left(\omega_{n}\right) \gamma_{\|, j}(\alpha) \gamma_{\|, j}(-\alpha)+\right. \\
& +\Delta_{\perp}^{-1}\left(\vec{q}, \omega_{n}\right) \cdot\left[\gamma_{\perp j}(\alpha)\left(\gamma_{\perp j}(\beta) M_{j}\left(\vec{q}, \omega_{n}\right)-\gamma_{\perp j}(-\beta) L_{j}\left(\vec{q}, \omega_{n}\right)\right)+\right. \\
& \left.\left.+\left(\alpha \rightarrow-\alpha ; \beta \rightarrow-\beta ; i \omega_{n} \rightarrow-i \omega_{n}\right)\right]\right\}
\end{aligned}
$$

where $G_{j \alpha(p, q)}(\omega)=\left[\omega+E_{j p}-E_{j q}\right]^{-1} ; b_{j \alpha(p, q)}=N_{j p}-N_{j q}$. In the low temperature limit the occupation numbers $N_{j p}$ of the quantum states $\left|\psi_{p ; j}\right\rangle$ are assumed to be $N_{j p}=1$ if $\left|\psi_{p ; j}\right\rangle$ is the ground state of eqn.(5), and $N_{j p}=0$ in other cases. The functions $M_{j}\left(\vec{q}, \omega_{n}\right)$ can be found analytically, but we do not present them here because they are cumbersome and unnecessary. 
The spectrum of elementary excitations of the system can be determined from finding the poles of the Green's functions, namely, from solving the equations

$$
\begin{aligned}
& \Delta_{\|}(\vec{q}, \omega)= 1-4 J_{q}^{2} u_{A}(\omega) u_{B}(\omega)=0 ; \\
& \Delta_{\perp}(\vec{q}, \omega)= {\left[1-\frac{1}{4} J_{q}^{2} z_{A}(\omega) z_{B}(\omega)\right]\left[1-\frac{1}{4} J_{q}^{2} z_{A}(-\omega) z_{B}(-\omega)\right]-\frac{J_{q}^{4}}{16} \times } \\
&\left(z_{A}(\omega) z_{A}(-\omega) w_{B}^{2}(\omega)+z_{B}(\omega) z_{B}(-\omega) w_{A}^{2}(\omega)-w_{A}^{2}(\omega) w_{B}^{2}(\omega)\right)- \\
&-\frac{1}{2} J_{q}^{2} w_{A}(\omega) w_{B}(\omega)=0 .
\end{aligned}
$$

The solutions of the equations $\Delta_{\|}(\vec{q}, \omega)=0$ and $\Delta_{\perp}(\vec{q}, \omega)=0$ determine the so-called longitudinal $\omega_{\|}(q, \omega)$ and transverse $\omega_{\perp}(q, \omega)$ branches of the magnonic spectrum, respectively. Recall, that in the case $\varphi=0$, these branches are determined by eqn.(3). Note also that for the isotropic magnet, the above-used decoupling of the Green's functions (the Hubbard-I approximation) gives results equivalent to the well-known results that the spin-wave theory obtained, for example, using the Tyablikov approximation [14]. For this reason, the functions describe the transverse and longitudinal spectra of spin waves. In this case the damping of magnonic quasiparticles is not taken into account. And finally note that the above procedure for obtaining the spin-wave excitation spectrum is of a universal character and can be used to study magnets with an arbitrary single-ion anisotropy.

The functions $u_{j}(\omega), z_{j}(\omega)$ and $w_{j}(\omega)$ included in the expression (11) are determined through the characteristics of the single-ion spectrum $E_{p}$, matrix elements $\gamma_{\perp j}(\alpha)$, and single-ion occupation numbers $N_{j, p}$ as follows $(j=A, B)$

$$
\begin{aligned}
u_{j}(\omega) & =\sum_{\alpha}\left|\gamma_{\| j}(\alpha)\right|^{2} G_{j \alpha}(\omega) b_{A}(\alpha) ; z_{j}(\omega)=\sum_{\alpha}\left|\gamma_{\perp j}(\alpha)\right|^{2} G_{j \alpha}(\omega) b_{j}(\alpha) ; \\
w_{j}(\omega) & =\sum_{\alpha} \gamma_{\perp j}(\alpha) \gamma_{\perp j}(-\alpha) G_{j \alpha}(\omega) b_{j}(\alpha) .
\end{aligned}
$$

From the direct calculations it can be shown that:

$$
u_{B}(\omega)=u_{A}(\omega) ; z_{B}(\omega)=z_{A}(\omega) ; w_{B}(\omega)=w_{A}(\omega) \cdot e^{2 i \varphi} .
$$

Thus, the longitudinal branches of spin-wave excitations do not change when the easy planes are rotated (when the angle $\varphi$ changes) and the transverse branches of the spectrum $\omega_{\perp}(q, \varphi)$ are of interest. In the case of $\varphi=0$ the functions $z_{A}(\omega), w_{A}(\omega)$ can be obtained analytically in the low temperature limit: [10]:

$$
z_{A}(\omega)=\frac{2 \varepsilon_{21}+2 \omega \cos 2 \theta}{(\omega)^{2}-\varepsilon_{21}^{2}} ; w_{A}(\omega)=\frac{2 \varepsilon_{21} \sin 2 \theta}{(\omega)^{2}-\varepsilon_{21}^{2}} ;
$$

Hence, the $\Delta_{\perp}(q, \omega)$ vs. $\varphi$ dependence can be expressed through the characteristics of the spectrum of a singlesublattice easy-plane magnet (the case of $\varphi=0$ ):

$$
\begin{aligned}
& \Delta_{\perp}(q, \omega, \varphi)=\Delta_{\perp}(q, \omega, \varphi=0)+J_{q}^{2} w_{A}^{2} \sin ^{2} \varphi \\
& \Delta_{\perp}(q, \omega, \varphi=0)=\frac{\left(\omega^{2}-\omega_{\perp}^{2}(q)\right)\left(\omega^{2}-\omega_{\perp}^{2}(q+\pi)\right)}{\left(\omega^{2}-\varepsilon_{21}^{2}\right)^{2}} .
\end{aligned}
$$

With the use of these expressions, we obtain the dispersion equation for the system under consideration with arbitrary value $\varphi$ (Fig. 1b):

$$
\Delta_{\perp}(q, \omega, \varphi)=\frac{\left(\omega^{2}-\omega_{\perp}^{2}(q)\right)\left(\omega^{2}-\omega_{\perp}^{2}(q+\pi)\right)+4 J_{q}^{2} \varepsilon_{21}^{2} \sin ^{2} 2 \theta \cdot \sin ^{2} \varphi}{\left(\omega^{2}-\varepsilon_{21}^{2}\right)^{2}}=0 .
$$

From the solution of this equation, the spin-wave spectrum vs. quasimomentum dependence has been obtained:

$$
\omega_{\perp ; 1,2}^{2}(q, \varphi)=\frac{1}{2}\left[\omega_{\perp}^{2}(q)+\omega_{\perp}^{2}(q+\pi) \pm \sqrt{\left(\omega_{\perp}^{2}(q)-\omega_{\perp}^{2}(q+\pi)\right)-16 J_{q}^{2} \varepsilon_{21}^{2} \sin ^{2} 2 \theta \cdot \sin ^{2} \varphi}\right] .
$$

In the case of mutually orthogonal easy planes $(\varphi=\pi / 2$, Fig. 1b), the expressions are simplified and take the form:

$$
\begin{aligned}
\omega_{\perp, 1}^{2}(q, \varphi=\pi / 2) & =\left(\varepsilon_{21}-J_{q} \cos ^{2} 2 \theta\right)^{2}=\omega_{\perp}^{2}(q)+\Delta_{q}^{2} ; \\
\omega_{\perp, 2}^{2}(q, \varphi=\pi / 2) & =\left(\varepsilon_{21}+J_{q} \cos ^{2} 2 \theta\right)^{2}=\omega_{\perp}^{2}(q+\pi)-\Delta_{q}^{2} ; \\
\Delta_{q}^{2} & =4 \varepsilon_{21} J_{q} \sin ^{2} \theta .
\end{aligned}
$$

The dependencies $\omega_{\perp, 1,2}(\varphi=\pi / 2)$ vs. $q$ are shown in Fig. 2 by the blue and green solid curves. Note that the branch $\omega_{\perp, 1}^{2}(q, \varphi=\pi / 2)$ should make the main contribution to the low temperature magnetic properties of the system. As 


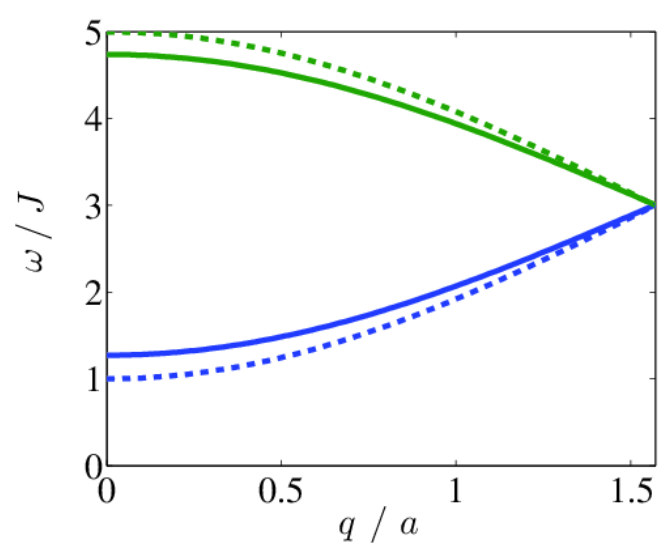

FIG. 2. Spin-wave spectra for 1D magnets with single-ion anisotropy of the easy plane and easy axis types. Solid and dashed lines corresponds to the $\omega_{\perp, 1,2}(\varphi=\pi / 2)$ and $\tilde{\omega}_{\perp ; 1,2}$ vs $q$ dependencies defined by the eqn.(16) $(D=J)$ and eqn.(20) $\left(D_{\text {eff }}=0.5 \cdot J\right)$, respectively.

can be seen from the Fig. 2, this branch is characterized by the excitation gap spectrum and by comparable to the value of this gap dispersion. Such features are characteristic of the excitations of easy-axis magnets.

In order to show the formation of an effective easy axis in the case of $\varphi=\pi / 2$ more formally, let us use that the dependence of the basic formulas (10)-(12) on the representation parameters (8) and single-ion energy spectrum $E_{p}$ are universal and does not depend on specific structure of the single-ion operators in (1). This allows, within the framework of the same approach, to consider two-sublattice 1D magnet with $S_{A, B}=1$ and easy axis single-ion anisotropy. Its Hamiltonian is the (1) with the single-ion terms in the form:

$$
\mathscr{H}_{A(B)}^{a}\left(\vec{S}_{f(g)}\right) \rightarrow \tilde{\mathscr{H}}_{A(B)}^{a}\left(\vec{S}_{f(g)}\right)=2 D_{e f f}\left(S_{f(g)}^{z}\right)^{2}-\tilde{H} S_{f(g)}^{z} ;
$$

where $\tilde{H}=2 J-H$. In this case we obtain the expressions for eigenfunctions and eigenvalues for the both sublattices:

$$
\left|\tilde{\psi}_{1,3}\right\rangle=| \pm 1\rangle ; \quad \tilde{E}_{1,3}=2 D_{\text {eff }} \mp \tilde{H} ;\left|\tilde{\psi}_{2}\right\rangle=|0\rangle ; \quad \tilde{E}_{2}=0 .
$$

Computations similar to the case of easy-plane anisotropy, but using the eigenstates (19) of single-ion Hamiltonians $\tilde{\mathscr{H}}_{A(B)}^{a}\left(\vec{S}_{f(g)}\right)$, make it possible to calculate the spin-wave spectrum of excitations of an easy-plane 1D magnet:

$$
\tilde{\omega}_{\perp, 1}^{2}(q, \pi / 2)=\left(2 D_{\text {eff }}+J-J_{q}\right)^{2} ; \tilde{\omega}_{\perp, 2}^{2}(q, \pi / 2)=\left(2 D_{\text {eff }}+J+J_{q}\right)^{2} .
$$

The dependencies $\tilde{\omega}_{\perp ; 1,2}$ vs. $q$ are shown in Fig. (2) by the blue and green dashed lines, respectively. It can be seen that the the spectra $\omega_{\perp, 1,2}(q, \varphi=\pi / 2)$ and $\tilde{\omega}_{\perp ; 1,2}(q)$ are very closed to each other. Thus the thermodynamic properties of the strongly anisotropic 1D magnet with twisted and mutually orthogonal easy-planes and easy-axis 1D magnet should be similar. This achieves the goal of this work.

a)

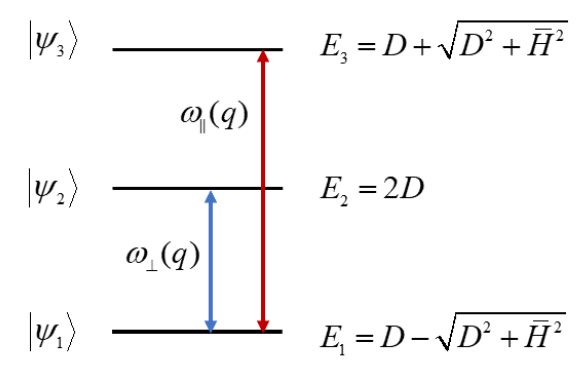

b)

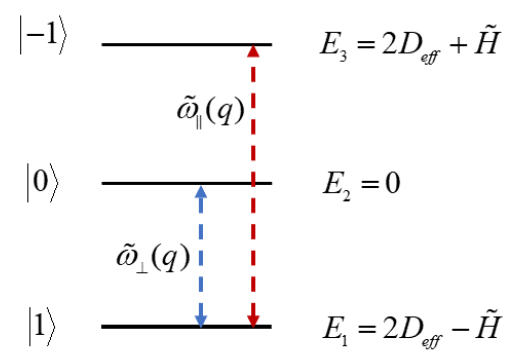

FIG. 3. The single-ion energy structure and quantum transitions responsible for the formation of quasiparticle bands of spin-wave excitations. a) the case of easy-plase magnet (eqns. (2), (5)). b) the case of easy-axis magnet (eqns. (18), (19)). 
Let us briefly consider some of the peculiarities and the physical meaning of the obtained branches of the spinwave spectrum. The expressions (11) and (12) show that the transverce $\omega_{\perp}(q)$ and longitudinal $\omega_{\|}(q)$ branches result from single-ion transitions with non-zero matrix elements of the operators $S^{+}$and $S^{z}$, respectively. The structures of the single-ion spectra of the easy-plane (eqns. (2) and (5)) and the easy-axis (eqns. (18) and (19)) magnets are shown in the Fig. 3a and Fig. 3b, respectively. For example, the transverse excitation branch $\omega_{\perp}(q)$ in easy-plane magnet results from the hybridization of single-ion quantum transitions between the states $\left|\psi_{1}\right\rangle=e^{-i \varphi} \cos \theta|1\rangle+e^{i \varphi} \sin \theta|-1\rangle$ and $\left|\psi_{2}\right\rangle=|0\rangle$ with the energies $E_{1}=D-\sqrt{D^{2}+\bar{H}^{2}}$ and $E_{2}=2 D$, respectively. In the case $\varphi=0$ we have the following spin-averages for the initial state $\left|\psi_{1}\right\rangle$ of such transition:

$$
\left\langle\psi_{1}\left|S^{x, y}\right| \psi_{1}\right\rangle=0 ;\left\langle\psi_{1}\left|\left(S^{x, y}\right)^{2}\right| \psi_{1}\right\rangle=\frac{1}{2}(1 \pm \sin 2 \theta) ;\left\langle\psi_{1}\left|S^{z}\right| \psi_{1}\right\rangle=\cos 2 \theta
$$

In the limit $D / J \gg 1$ such averages correspond to the spins lying in the easy plane. For the final state of the transition $\left|\psi_{2}\right\rangle$ we have $\left\langle\psi_{2}|\vec{S}| \psi_{2}\right\rangle=\left\langle\psi_{2}\left|\left(S^{z}\right)^{2}\right| \psi_{2}\right\rangle=0 ;\left\langle\psi_{2}\left|\left(S^{x, y}\right)^{2}\right| \psi_{2}\right\rangle=1$. Thus in strongly anisotropic limit the quantum transition $\left|\psi_{1}\right\rangle \rightarrow\left|\psi_{2}\right\rangle$ can be qualitatively considered as a spin flop from the easy plane into a plane perpendicular to the $z$-axis. In the limit of noninteracting ions, the single-ion transitions form highly degenerate levels, but when the exchange interaction between the sublattices is taken into account (the last term of the eqn.1), such transitions hybridize and form bands with the dependence of the excitation energy on the quasimomentum. The actual for this work single-ion transitions are shown by vertical lines with arrows. The corresponding quasi-momentum dependences of the spin-wave bands are depicted near the vertical lines. Note, that dependences $\omega_{\perp}(q)$ and $\tilde{\omega}_{\perp}(q)$ in Fig. 3 correspond to the low-lying quasiparticle branches shown in Fig. 2.

Note that in this work, the calculations were carried out under the assumption that at zero temperatures only the ground single-ion states are filled: $N_{j ; 1}=1 ; N_{j ; 2,3}=0(j=A, B)$. In other words, we have neglected the quantum fluctuations due to spin-wave renormalizations. To take such effects into account, the single-ion occupation numbers should be calculated using the spectral theorem [14] by the formula:

$$
N_{j p}=\frac{1}{2} \sum_{r} \int_{-\pi / 2}^{\pi / 2} d q\left[\frac{\left(F_{j}\left(\alpha, q, \omega_{r}\right)+F_{j}\left(\alpha, q,-\omega_{r}\right)\right) f_{B}\left(\frac{\omega_{r}}{T}\right)}{\omega_{r} \prod_{l \neq r}\left(\omega_{l}^{2}-\omega_{r}^{2}\right)}+\frac{F_{j}\left(\alpha, q,-\omega_{r}\right)}{\omega_{r} \prod_{l \neq r}\left(\omega_{l}^{2}-\omega_{r}^{2}\right)}\right] .
$$

Moreover, the calculation of the $N_{j p}$ should be self-consistent with the calculation of the Green's functions (10). In the (22) the last ones has been represented in the form:

$$
G_{j \alpha ; j \alpha}(q, \omega)=\frac{F_{j}\left(\alpha, q, \omega_{k}(q)\right)}{\prod_{k}\left(\omega^{2}-\omega_{k}^{2}(q)\right)} .
$$

In this approximation, the energy spectrum and physical observables will be renormalized. However, such consideration is beyond the scope of this work.

\section{Conclusion}

In this work, on the basis of analytical solutions for the excitation spectra of simple 1D spin-models, we have demonstrated the effect of formation of effective easy axis in strongly anisotropic easy-plane magnets with several sublattices.

This effect is due to the different orientations of the easy planes for different sublattices. This result clarifies the lowtemperature behavior of the anisotropic metal-organic single chain magnet catena $-\left[\mathrm{Fe}^{I I}\left(\mathrm{ClO}_{4}\right)_{2} \mathrm{Fe}^{I I I}(\text { bpca })_{2}\right]\left(\mathrm{ClO}_{4}\right)$.

\section{Acknowledgements}

S. M. S. expresses deep gratitude to V. V. Val'kov. The main results of this work were with substantial use of the theory developed by him (see for example articles [10], [12] and references given in them), as well as on his previous advice. S. M.S. and M.N.P. acknowledges the support from the Foundation for the Advancement of Theoretical Physics and Mathematics "BASIS" (Grants No. 20-1-4-25-1 and 19-1-1-12-2, respectively).

\section{References}

[1] Zhang W.-X., Ishikawa R., Breedlove B., Yamashita M. Single-chain magnets: beyond the Glauber model. RSC Advances, 2013, 3, P. 37723798.

[2] Smirnov A.I., Glazkov V.N. Mesoscopic spin clusters, phase separation, and induced order in spin-gap magnets: A review. Journal of Experimental and Theoretical Physics, 2007, 105, P. 861-879.

[3] Kosmachev O.A., Fridman Yu.A., Galkina E.G., Ivanov B.A. Dynamic properties of magnets with spin S = 3/2 and non-Heisenberg isotropic interaction. Journal of Experimental and Theoretical Physics, 2015, 147, P. 320.

[4] Kudasov Yu.B., Korshunov A.S., Pavlov V.N., Maslov D.A. Frustrated lattices of Ising chains. Physics-Uspekhi, 2012,55 , P. $1169-1191$. 
[5] Billoni O.V., Pianet V., Pescia A. V. Static and dynamic properties of single-chain magnets with sharp and broad domain walls. Physical Review B, 2011, 84, P. 064415(1-16).

[6] Liu T., Zhang Y.-J., Kanegawa S., Sato O. Photoinduced Metal-to-Metal Charge Transfer toward Single-Chain Magnet. Journal of the American Chemical Society, 2010, 132, P. 8250-8251.

[7] Val'kov, V. V., Shustin, M. S. Quantum Theory of Strongly Anisotropic Two-and Four-Sublattice Single-Chain Magnets. Journal of Low Temperature Physics, 2016, 185, P. 564-568.

[8] Kajiwara T., Tanaka H., Yamashita M. Single-chain magnets constructed with a twisting arrangement of the easy-plane of iron (II) ions. Pure and Applied Chemistry, 2008, 80, P. 2297.

[9] Kajiwara T., Tanaka H., Nakano M., Takaishi Sh., Nakazawa Ya., Yamashita M. Single-Chain Magnets Constructed by Using the Strict Orthogonality of Easy-Planes: Use of Structural Flexibility to Control the Magnetic Properties. Inorganic Chemistry, 2010, 49, P. 8358

[10] Val'kov V.V., Val'kova T.A. Low-temperature magnetization of an easy-plane ferromagnet. Low Temperature Physics, 1985, 11 (9), P. 951959.

[11] Fridman Yu.A., Kosmachev O.A. Quantum effects in an anisotropic ferrimagnet. Physics of Solid State, $2009, \mathbf{5 1}$, P. 1167.

[12] Val'kov, V. V., Shustin, M. S. Quantum renormalizations in anisotropic multisublattice magnets and the modification of magnetic susceptibility under irradiation. Journal of Experimental and Theoretical Physics, 2015, 121, P. 860.

[13] Zaitcev R.O. Generalized diagram technique and spin waves in anisotropic antiferromagnet. Soviet Journal of Experimental and Theoretical Physics, 1975, 68, P. 207 (Ru).

[14] S.V. Tyablikov, Methods in the Quantum Theory of Magnetism. Nauka, Moskow, 1975.

[15] J.Hubbard, J. Proc. Roy. Soc. A, 1963, 276, P. 238. 